\title{
Psicooncología
}

ISSN: 1696-7240

\section{Adaptación española de la Escala de Control Personal Percibido (Perceived Personal Control) en Consejo Genético}

\author{
Melinda González-Concepción ${ }^{1, *}$; Vanesa Castejón ${ }^{2}$; Ignacio Blanco ${ }^{3}$; Tomás Blasco ${ }^{4}$
}

Recibido: 11 de septiembre de 2017 / Aceptado: 15 de enero de 2018

Resumen. Objetivo: Analizar la validez y la fiabilidad de la adaptación de la escala Perceived Personal Control (PPC) en el contexto español para pacientes portadoras de mutación en los genes $B R C A 1 / 2$ responsables del cáncer de mama y ovario hereditario (CMOH). Método: Adaptación transcultural y validación de la escala Perceived Personal Control (PPC) desarrollada por Shiloh y colaboraradores mediante traducción, retrotraducción y validación a través de un análisis factorial exploratorio con rotación Oblimin en una muestra de 176 mujeres portadoras de genes BRCA 1/2 para CMOH. Resultados: La versión española de la PPC reduce a seis los nueve ítems de la escala original, dado que esta estructura es la que ofrece una solución factorial más satisfactoria. El análisis factorial mostró un solo factor que explica el 51,07\% de la varianza, en el que todos los ítems tenían cargas factoriales por encima de 0,4. El coeficiente $\alpha$ de Cronbach fue de 0,84 para el conjunto de la escala, la cual permite obtener valores que oscilan entre 0 (bajo grado de percepción de control) y 2 (alto grado de percepción de control). Conclusiones: La adaptación española de la Escala de Percepción de Control (PPC6) posee propiedades psicométricas satisfactorias en la versión de 6 ítems con un solo factor, por lo que su utilización en contexto español Consejo Genético para cáncer hereditario parece adecuada.

Palabras clave: Consejo genético; cáncer hereditario; percepción de control; cáncer de mama; cáncer de ovario.

\section{[en] Spanish adaptation of the Perceived Personal Control (PPC) in genetic counseling}

Abstract. Purpose: The aim of this study was to analyze the reliability and validity of the spanish adaptation of the Perceived Personal Control (PPC) scale in women who were positive for BRCA1/2 genes, which are related with hereditary ovarian and breast cancer (HOBC). Method: PPC original items developed by Shiloh and co-workers were translated and back-translated in order to develop an spanish version which was analyzed by an exploratory factor analysis with Oblimin rotation. Answers to the spanish version were provided by a sample of 176 women who were positive for BRCA1/2

1 Melinda González. Unidad de melanoma, Hospital Clinic Barcelona (IDIBAPS), Escola Universitària d'Infermeria Terrassa (EUIT).

E-mail: melindagonzalez@euit.fdsll.cat

2 Vanesa Castejón. Institut Català d’Oncologia (ICO), Hospital Duran i Reynals, Barcelona

E-mail: vanepsico@hotmail.com

3 Ignacio Blanco. Programa de Asesoramiento y Genética Clínica. Institut Català de la Salut. Hospital Universitari Germans Trias i Pujol

E-mail: ignacio.blanco.guillermo@gmail.com

4 Tomás Blasco, Unidad de Psicologia Básica. Universitat Autònoma de Barcelona.

E-mail: tomas.blasco@uab.es

* Dirección de correspondencia: Melinda González-Concepción. Unidad de melanoma, Hospital Clinic Barcelona (IDIBAPS), Escola Universitària d’Infermeria Terrassa (EUIT). E-mail: melindagonzalez@euit.fdsll.cat 
for HOBC. Results: Spanish version contains only six items of the nine original items, since this structure provided the optimal solution to the exploratory factor analysis. This version is a one-factor scale $(51.07 \%$ of variance explained) and all items had factor loading values $>0.4$. The scale has good reliability (Cronbach's Alpha $=0.84$ ) and gives a range of values between 0 (low perceived control) and 2 (high perceived control). Conclusions: Spanish adaptation (PPC6) of the original 9-items version has enough reliability and psychometric properties, and it seems to be useful to be applied in Genetic Counseling at spanish cultural settings.

Keywords: Genetic counseling; hereditary cancer; perceived control; breast cancer; ovarian cancer.

Sumario. 1. Introducción 2. Método 2.1. Participantes 2.2. Material 2.3. Procedimiento 2.4. Aspectos éticos 3. Análisis estadístico 4. Resultados 5. Discusión 6. Agradecimientos 7. Referencias bibliográficas.

Cómo citar: González-Concepción M, Castejón V, Blanco I, Blasco T. Adaptación española de la Escala de Control Personal Percibido (Perceived Personal Control) en Consejo Genético. Psicooncología 2018;15:23-36. Doi: 10.5209/PSIC.59172.

\section{Introducción}

El cáncer es una enfermedad compleja y multifactorial, donde intervienen factores ambientales y genéticos. El cáncer puede considerarse una enfermedad genética, dado que la transformación neoplásica de las células tumorales se produce por la adquisición de mutaciones en diferentes genes. Sin embargo, estas mutaciones se producen generalmente en células somáticas que no participan en la generación de un nuevo ser por lo que, aún siendo una enfermedad genética en absoluto quiere decir que siempre sea hereditaria ${ }^{(1)}$. Más bien al contrario, sólo entre un 5-10\% de los tumores son en realidad hereditarios ${ }^{(1)}$.

La identificación de las alteraciones genéticas implicadas en el desarrollo del cáncer de mama tanto esporádico como hereditario está permitiendo individualizar las medidas de tratamiento y prevención. Hasta la fecha, para determinar el riesgo de padecer cáncer de mama y ovario se valoraban factores como la historia personal, la edad y el sexo. En la actualidad se ha demostrado la importancia de incluir también la historia familiar. Además, la identificación de genes de alta penetrancia que predisponen al cáncer de mama y ovario hereditario, como son los genes BRCA1 y BRCA2, permite detectar a mujeres sanas que presentan un elevado riesgo de desarrollar cáncer. Es posible establecer entonces estrategias personalizadas de prevención o tratamiento con la intención de modificar la historia natural de estas personas ${ }^{(2)}$.

Sin embargo, este avance médico que permite individualizar el modo de abordar la prevención, implica que las pacientes tienen que gestionar un concepto difícil como es el manejo del riesgo de desarrollar una enfermedad grave, lo que puede generar un impacto emocional cuyo manejo puede requerir de un soporte psicológico ${ }^{(3)}$. Así, el proceso de consejo genético puede ser vivido como una situación amenazante generadora de estrés provocando un malestar emocional importante, tal y como ha quedado constatado en diferentes trabajos ${ }^{(3)}$.

Este impacto emocional aparece sobre todo en el momento en que la persona comienza el proceso de Consejo Genético, y evoluciona a lo largo del mismo, sugiriendo la literatura que, a medida que pasa el tiempo, este impacto emocional, en muchos casos, se atenúa y la persona continúa sus visitas y controles con unos bajos 
niveles de estrés emocional ${ }^{(4,5)}$. La evolución del impacto emocional determina en buena medida la adaptación de la persona al proceso de Consejo Genético y corresponde a los equipos asistenciales lograr que ese impacto emocional se maneje de la manera más eficaz posible a fin de conseguir que el paciente se incorpore a los protocolos aplicables en su caso y los lleve a cabo de manera correcta, evitándose así el abandono o un uso inadecuado de los recursos sanitarios, los cuales imposibilitarían conseguir los resultados que el Consejo Genético ofrece.

Es por ello que se han dedicado estudios a conocer los factores que determinan el impacto emocional y su evolución, a fin de facilitar a las unidades de Consejo Genético claves útiles para identificar a las pacientes que pueden necesitar más estrategias de soporte para seguir adelante con los protocolos ${ }^{(6,7)}$, así como también para facilitar que las personas movilicen los recursos que ya poseen (como son las estrategias de afrontamiento) $)^{(8,9)}$ y que les permitirán manejar la situación de forma eficaz.

Es crucial que los equipos de Consejo Genético puedan manejar el impacto emocional en el momento del inicio del proceso, pero también a lo largo del mismo en los casos de aquellas pacientes en los que este impacto no se reduce a lo largo del tiempo. De entre las diversas variables identificadas por la literatura, hay dos que destacan por cuanto recogen los elementos fundamentales que el proceso de Consejo Genético quiere transmitir a la paciente, y que no es otro que la posibilidad de ejercer un cierto control sobre el riesgo de aparición del cáncer. El primer elemento es la comprensión, por parte de la paciente, de que se encuentra en una situación de riesgo superior al del resto de la población. El segundo elemento es que la paciente sepa identificar tanto el riesgo existente como las posibilidades de control que se pueden desarrollar. Estos elementos constituyen la base que permitirá manejar mejor el impacto emocional y, a la vez, facilitar el seguimiento de los protocolos.

Respecto a la percepción de riesgo, los estudios señalan que las estimaciones del riesgo de padecer cáncer pueden mejorar tras el consejo genético ${ }^{(10)}$; es decir: el riesgo objetivo y el riesgo percibido tienden a coincidir. Sin embargo, es frecuente que esas estimaciones sigan siendo imprecisas ${ }^{(11,12)}$.

Si bien la percepción de riesgo y la percepción de control son elementos que pueden interactuar entre sí, algunos modelos de adaptación a la enfermedad así como diversas investigaciones que han puesto de manifiesto la relación entre percepción de control y adaptación al cáncer ${ }^{(13)} \mathrm{y}$ a otras enfermedades ${ }^{(14,15)}$ consideran que es la percepción de control el elemento fundamental que va a permitir hacer frente a la enfermedad. Dentro de esta línea, y aplicándola al ámbito del Consejo Genético se encuentran los trabajos desarrollados por el grupo de Shiloh et al. ${ }^{(16)}$.

Shiloh y su equipo proponen un modelo que establece la percepción de control como variable mediadora en el afrontamiento del proceso de Consejo Genético. Concretamente, este Control Personal Percibido (Perceived Personal Control, $P P C)$, por sus siglas en inglés, se estructura en tres dimensiones: una dimensión cognitiva, una dimensión conductual, y una dimensión decisional. A partir de aquí elaboran una escala (Escala PPC) que consta de 9 ítems ${ }^{(16)}$ y que integra esa estructura tridimensional.

Puede parecer que los trabajos de Shiloh permiten disponer de un instrumento adecuado para el análisis de la percepción de control en el contexto del Consejo Genético. Sin embargo, autores que han seguido su línea no han encontrado siempre 
esta estructura tridimensional, y algunos señalan que la percepción de control es, al menos en el caso del Consejo Genético, un constructo unidimensional. No está clara la razón por la que los diversos autores hallan esas tres dimensiones o sólo una para la PPC. Algunos trabajos ${ }^{(17,18)}$ asumen el modelo tridimensional sin contrastarlo a través del análisis factorial (cosa que sí hicieron Shiloh y su grupo en el trabajo original de 1997), siendo los trabajos que sí utilizan este análisis ${ }^{(19,20)}$ los que proponen visiones alternativas que llevan a plantear la existencia de un solo factor. Es posible que las diferencias culturales permitan entender estas discrepancias, ya que el trabajo original de Shiloh y su equipo ${ }^{(16)}$ se llevó a cabo en población judía, mientras que los estudios que proponen un modelo unidimensional han evaluado a pacientes que no pertenecen a esta comunidad.

Dada la importancia que reviste la percepción de control dentro del proceso de Consejo Genético como elemento que puede permitir a los equipos asistenciales facilitar una mejor atención a los pacientes, es pertinente disponer de un instrumento de evaluación de dicha percepción. Puesto que no conocemos la existencia, dentro de nuestro ámbito cultural, de un instrumento de estas características, hemos considerado necesario llevar a cabo una adaptación española del instrumento propuesto por Shiloh $\mathrm{y}$, a la vez, aportar elementos que permitan dilucidar si la percepción de control es un concepto uni o multidimensional. En consecuencia, el objetivo de nuestro trabajo es el de elaborar una adaptación del instrumento PPC y analizar la validez y fiabilidad de la misma en el contexto cultural de nuestro país.

\section{Método}

\subsection{Participantes}

Las participantes en esta investigación formaban parte de un estudio más amplio destinado a valorar la experiencia de mujeres portadoras de genes de alta penetrancia para el cáncer de mama y/o ovario (BRCA1/2). Todas ellas estaban atendidas en el Programa de Cáncer Hereditario del Institut Catala d'Oncologia y recibieron visita de seguimiento entre los meses de julio de 2012 y mayo de 2014 en los diferentes centros en los que se lleva a cabo el programa.

Los criterios de inclusión para participar en el estudio fueron:

o Ser portadora de $B R C A 1$ o $B R C A 2$ afecta (esto es, haber sido diagnosticada de cáncer de mama y/o ovario), o bien ser portadora sana (esto es, $\underline{n o}$ haber sido diagnosticada de cáncer de mama y/o ovario, pero sí ser portadora de mutación en el gen BRCA1 o BRCA2). Estas pacientes serán identificadas como PA (portadoras afectas) y PS (portadoras sanas, no afectas), respectivamente.

o Ser mayores de 18 años.

o No ser analfabetas.

o No tener enfermedad mental o limitación cognitiva.

o Entender el español.

Un total de 176 mujeres participaron en el estudio, 98 de las cuales $(55,7 \%)$ son portadoras afectas (PA).

La edad media de las portadoras sanas (PS) es de 41,12 años, y la edad media de las PA es de 52,96, siendo esta diferencia estadísticamente significativa $(\mathrm{t}=6,52$; 
$\mathrm{p}=0,00)$. Hay también diferencias estadísticamente significativas entre PA y PS en el hecho de tener hijos (chi-cuadrado $=5,15 ; \mathrm{p}=0,023$ ), ya que el porcentaje de las primeras que tiene descendencia es superior $(84,7 \%$ vs. $70,5 \%)$. Ambas diferencias pueden explicarse por el hecho de que tener un tumor es algo que aparece más tarde en el ciclo vital de la persona y, además, que al tener las pacientes PA una edad más avanzada, ésta conlleva una mayor probabilidad de tener hijos

\subsection{Material}

La escala del Control Personal Percibido (Perceived Personal Control; PPC) fue elaborada y validada por el grupo encabezado por Shiloh ${ }^{(16,21)}$ para detectar la percepción de control que tenían los pacientes que acudían a consejo genético no específicamente oncológico. La escala original está formada por 9 ítems, donde los autores proponen una estructura factorial de 3 factores. Cada ítem es evaluado mediante una escala de respuesta tipo Likert con puntuación entre 0 y 2 . Puntuaciones altas indican alto grado de percepción de control.

El proceso de adaptación transcultural de la escala se realizó siguiendo los protocolos propuestos por Cha, Kim y Erlen ${ }^{(22)}$ y Chen y Boore ${ }^{(23)}$. Así, la traducción y adaptación de la versión inglesa de las escalas se realizó por 4 personas bilingües (lingüistas y especialistas en cáncer). Las versiones resultantes de las dos escalas se evaluaron en los 5 niveles de equivalencia deseados (contenido, sintáctica, técnica, criterio y conceptual). Cada uno de los ítems se calificó como A (totalmente equivalente), B (bastante equivalente) y C (equivalencia dudosa) y se realizó una reunión de consenso del equipo investigador para redactar una versión preliminar de las escalas en español.

En la tabla 1 se indican los ítems originales de la versión en inglés y el factor al que pertenecen, y la traducción asumida por el equipo investigador.

Tabla 1. Ítems originales de la escala PPC y su equivalencia en español

\section{Ítem original}

I think I understand what problem brought me to genetic counselling a

I feel I know the meaning of the problem for my family's future and me ${ }^{\text {a }}$

I know what caused the problem ${ }^{\text {a }}$

I feel I have the tools to make decisions that will influence my future ${ }^{b}$

I feel I can make a logical evaluation of the various options available to me in order to choose one of them ${ }^{b}$

I feel I can make decisions that will change my family's future ${ }^{\mathrm{b}}$

I feel there are certain things I can do to prevent the problem from recurring ${ }^{c}$

I feel I know what to do to ease the situation

I think I know what should be my next steps

\section{Ítem Adaptado Español}

Creo que entiendo el problema que me llevó a Consejo Genético.

Siento que conozco el significado del problema para mi futuro y el de mi familia.

Creo que sé lo que causó el problema.

Siento que tengo las herramientas necesarias para tomar decisiones que influirán en mi futuro.

Siento que puedo hacer una evaluación lógica de las diversas opciones disponibles para mí con el fin de elegir una de ellas.

Siento que puedo tomar decisiones que cambiarán el futuro de mi familia.

Creo que hay ciertas cosas que puedo hacer para evitar que el problema vuelva a ocurrir.

Siento que sé qué hacer para aliviar la situación.

Creo que conozco los próximos pasos que tengo que seguir.
Clasificación

a. Control cognitivo. b. Control decisional. c. Control conductual 


\subsection{Procedimiento}

Una vez finalizada la visita de seguimiento que las pacientes tenían programada en la unidad, el médico responsable, la enfermera, o la investigadora (primera autora del presente trabajo) explicaban a la paciente que se estaba llevando a cabo un estudio destinado a conocer cómo afecta a las personas el proceso de Consejo Genético y en qué medida les ayuda el asesoramiento que proporciona el personal de la Unidad de Consejo Genético. Para ello se solicitaba su colaboración para rellenar una batería de cuestionarios que se les entregaba para que la rellenasen en su domicilio. El cuestionario PPC formaba parte de esa batería, en la que se incluían otros elementos que no forman parte de la presente investigación.

Una vez aceptaban se les proporcionaba un documento que constaba de la siguiente documentación:

- Hoja informativa.

- Consentimiento de participación.

- Un protocolo con los diferentes cuestionarios.

- Un sobre franqueado para la devolución de los mismos.

- Un teléfono de contacto y una persona de referencia.

Con la documentación presente se leía la carta informativa en voz alta, destacando los objetivos del estudio, y la confidencialidad de los datos aportados. Se indicaba a la paciente que en cualquier momento podía dejar de participar en la investigación sin tener que dar explicaciones. A continuación, se facilitaba el formulario de consentimiento informado. Una vez firmado, se entregaba la batería de cuestionarios y se explicaba en qué consistía el procedimiento de devolución mediante el uso del sobre franqueado con la dirección de la unidad y hospital de referencia donde realizaban el seguimiento. El tiempo que necesitaban las pacientes para rellenar el cuestionario (que, como hemos señalado, incluía la escala PPC y otros elementos que no se consideran en la presente investigación) se estimaba en 45 minutos. Por este motivo se les explicaba que podían contestar en diferentes momentos si se encontraban muy cansadas. Se les facilitaba un número de teléfono para que pudiesen contactar con el equipo investigador de lunes a viernes de 9-17 horas, si bien cabe señalar que no hubo contactos telefónicos para aclarar dudas respecto a cómo cumplimentar la batería de cuestionarios.

La batería de cuestionarios fue entregada a 329 pacientes entre los meses de Julio del 2012 y Mayo del 2014. En el seguimiento de las devoluciones, si la investigadora no recibía el cuestionario en un plazo 2-3 semanas posteriores a la fecha prevista, realizaba una llamada telefónica a la paciente (llamada de rescate) para intentar conocer el motivo por el cual no se había cumplimentado la batería de cuestionarios, a la vez que recordaba la importancia que tenía para el equipo poder disponer de los datos, pues eso permitiría conocer si los pacientes entendían la información proporcionada. Un total de 78 pacientes portadoras sanas de $B R C A 1 / 2$ y 98 portadoras afectas de $B R C A 1 / 2(53,49 \%$ del total) contestaron al estudio en el plazo previsto, sin necesidad de tener que hacer una llamada de rescate, y son los que constituyen la presente investigación. 


\subsection{Aspectos éticos}

El proyecto de investigación estaba autorizado por la Comisión de Ética de L'Intitut Investigació Biomedica de Bellvitge (IDIBELL). Para preservar la confidencialidad de los datos se asignó a cada individuo un código único. Este código estaba ubicado en la carta de presentación. La base de datos tenía una variable de identificación codificada de acceso restringido. El análisis estadístico se realizó sobre datos individuales carentes de información que permitiese la identificación de los participantes.

\section{Análisis estadístico}

Los datos se analizaron mediante el paquete estadístico SPSS para Windows 20.0 (IBM).

Las respuestas a la escala PPC se analizaron utilizando el Análisis Factorial Exploratorio (AFE). Se utilizó el método de mínimos cuadrados generalizados (GLS) con rotación Oblimin. Previamente se calculó el coeficiente Kaiser-MeyerOlkin (KMO) con el fin de verificar la idoneidad de la matriz de datos para el análisis factorial. Siguiendo el procedimiento de Beranuy et al. ${ }^{(24),}$ el criterio para considerar la adscripción del ítem a un factor estableció que la carga factorial fuera superior a 0.40. La fiabilidad de las escalas se calculó con el estadístico alfa de Cronbach y se consideró adecuada el valor igual o superior a 0,7 .

\section{Resultados}

Para el conjunto de los 9 ítems, la Matriz Factorial obtenida se indica en la tabla 2. Puede observarse que hay 6 ítems que cumplen el criterio de tener un valor de carga factorial superior a 0,4 (ítems 4 a 9). La varianza total explicada es del 43,35\%. Dado que los ítems 1 y 2 tenían unas cargas factoriales muy bajas, se decidió repetir el análisis factorial retirando dichos ítems.

En este segundo análisis, la varianza explicada es del 45,72\% y la solución factorial arroja un solo factor, tal y como muestra la Tabla 3. Puede observarse que el ítem 3 no alcanza la carga factorial exigida por nuestro criterio para formar parte del factor, por lo que se optó por realizar un tercer análisis factorial excluyendo este ítem.

El resultado del tercer análisis factorial (ver Tabla 4) indicó que el porcentaje de varianza explicada era del 51,07\% y todos los ítems tenían cargas factoriales por encima del valor 0,4 exigido, siendo, por tanto, una solución satisfactoria. Como criterio adicional se calculó el ratio chi-cuadrado/grados de libertad que dio un valor de 4,5 (chi cuadrado $=40,551 ; \mathrm{gl}=9$ ), el cual, de acuerdo con lo establecido en otros estudios que también utilizaron el análisis factorial exploratorio ${ }^{(24)}$ pudimos considerarlo como aceptable. Asimismo, el índice KMO dio un valor satisfactorio, próximo a $1(0,813)$ y la prueba de esfericidad de Bartlett dio hipótesis nula (chi cuadrado $=423,42 ; \mathrm{gl}=15 ; \mathrm{p}=, 000)$. Finalmente, el alfa de Cronbach también mostró un valor adecuado de 0,84 . 
Tabla 2. Matriz factorial descriptiva de los 9 ítems y sus pesos factoriales

\begin{tabular}{lccc}
\hline & \multicolumn{2}{c}{ Factor } \\
& 1 & 2 \\
\hline $\begin{array}{l}\text { PPC1. Creo que entiendo el problema que me llevó a Consejo Genético } \\
\begin{array}{l}\text { PPC2. Siento que conozco el significado del problema para mi futuro y } \\
\text { el de mi familia. }\end{array}\end{array}$ &, 280 &, 040 \\
$\begin{array}{l}\text { PPC3. Creo que sé lo que causó el problema. } \\
\begin{array}{l}\text { PPC4.Siento que tengo las herramientas necesarias para tomar decisio- } \\
\text { nes que influirán en mi futuro. }\end{array}\end{array}$ &, 683 &, 341 \\
$\begin{array}{l}\text { PPC5. Siento que puedo hacer una evaluación lógica de las diversas } \\
\text { opciones disponibles para mí con el fin de elegir una de ellas. }\end{array}$ &, 999 &,- 004 \\
$\begin{array}{l}\text { PPC6. Siento que puedo tomar decisiones que cambiarán el futuro de } \\
\text { mi familia. }\end{array}$ &, 465 &, 305 \\
$\begin{array}{l}\text { PPC7. Creo que hay ciertas cosas que puedo hacer para evitar que el } \\
\text { problema vuelva a ocurrir. }\end{array}$ &, 412 &, 458 \\
$\begin{array}{l}\text { PPC8. Siento que sé qué hacer para aliviar la situación. } \\
\text { PPC9. Creo que conozco los próximos pasos que tengo que seguir. }\end{array}$ &, 490 &, 732 \\
\hline
\end{tabular}

Tabla 3. Matriz factorial descriptiva de los 7 ítems y sus pesos factoriales.

\begin{tabular}{lc}
\hline & Factor \\
& 1 \\
\hline PPC3. Creo que sé lo que causó el problema. &, 363 \\
$\begin{array}{l}\text { PPC4. Siento que tengo las herramientas necesarias para tomar decisiones que } \\
\text { influirán en mi futuro. }\end{array}$ &, 770 \\
$\begin{array}{l}\text { PPC5. Siento que puedo hacer una evaluación lógica de las diversas opciones } \\
\text { disponibles para mí con el fin de elegir una de ellas. }\end{array}$ &, 830 \\
$\begin{array}{l}\text { PPC6. Siento que puedo tomar decisiones que cambiarán el futuro de mi familia. } \\
\text { PPC7. Creo que hay ciertas cosas que puedo hacer para evitar que el problema } \\
\text { vuelva a ocurrir. }\end{array}$ &, 581 \\
$\begin{array}{l}\text { PPC8. Siento que sé qué hacer para aliviar la situación. } \\
\text { PPC9. Creo que conozco los próximos pasos que tengo que seguir. }\end{array}$ &, 730 \\
\hline
\end{tabular}


Tabla 4. Matriz factorial descriptiva de los 6 ítems y sus pesos factoriales

Factor

1

PPC4. Siento que tengo las herramientas necesarias para tomar decisiones que , 774 influirán en mi futuro.

PPC5. Siento que puedo hacer una evaluación lógica de las diversas opciones , 836 disponibles para mí con el fin de elegir una de ellas.

PPC6. Siento que puedo tomar decisiones que cambiarán el futuro de mi familia.

PPC7. Creo que hay ciertas cosas que puedo hacer para evitar que el problema vuelva a ocurrir.

PPC8. Siento que sé qué hacer para aliviar la situación.

PPC9. Creo que conozco los próximos pasos que tengo que seguir.

\section{Discusión}

En este trabajo hemos realizado la adaptación transcultural de la escala PPC y el análisis de validez de constructo de la misma. Esta versión en español de la escala de percepción de control (PPC) en Consejo Genético para cáncer de mama y ovario hereditario, a diferencia de lo que plantearon los autores de la escala original, consta de un solo factor que puede evaluarse mediante seis ítems que proporcionan una fiabilidad satisfactoria.

Como se ha señalado en la introducción, no somos los únicos que hemos hallado una estructura unifactorial en la PPC. Diversos autores han realizado un análisis factorial exploratorio llegando a la solución de tres factores ${ }^{(16,21)}$ en contraposición de otros que pese a utilizar el mismo método se decantan por la solución unifactorial $^{(19,20)}$. Así, Shiloh et al. ${ }^{(16)}$ diseñan la escala en dos lenguas la hebrea y la inglesa. Para la versión hebrea llevan a cabo un análisis factorial exploratorio del que extraen los tres factores establecidos desde el marco teórico, pero advierten que se deberán aportar más datos en cuanto a las propiedades psicométricas de dicha escala en futuros trabajos. En este estudio no se desarrollaron las propiedades psicométricas de la versión inglesa y no es hasta dos años más tarde que el mismo equipo $^{(21)}$, decide explorar qué sucede a nivel psicométrico para dicha escala y lleva a cabo el análisis factorial confirmatorio (AFC) donde llega a la conclusión de que existían tres factores predeterminados que se ajustaban al modelo teórico propuesto por Averill ${ }^{(25)}$ siendo estos el control conductual, el control decisional y el control cognitivo respectivamente. La fiabilidad de la escala previa al proceso de consejo genético presentaba un alfa de Cronbach de 0,83 que se incrementaba hasta 0,86 cuando el paciente había recibido el asesoramiento correspondiente ${ }^{(21)}$.

Sin embargo, Smets et al. ${ }^{(19)}$ en Holanda, realizaron un análisis factorial confirmatorio del que concluyen que los tres factores obtenidos no se ajustaban a los contenidos que, en base al enfoque teórico, se esperaban (control cognitivo, control decisional, 
y control conductual) por lo que optaron finalmente por un análisis exploratorio que condujo a la solución de un solo factor que explicaba entre el 39\% y un $43 \%$ de la varianza. Smets et al. establecieron como criterio que para que un ítem formara parte de un factor éste debería tener una carga factorial no inferior a 0,30 . Todos los ítems cumplían este criterio y la escala que ellos propusieron en versión holandesa mantenía los 9 ítems originales. En cuanto a la fiabilidad obtenida, fue de 0,81 en pacientes que acudían a consejo genético por cáncer ${ }^{(19)}$. Dicha fiabilidad se mantuvo entre 0,70 y 0,85 en estudios posteriores que asumieron la estructura unifactorial de la $\operatorname{PPC}^{(10,26,27)}$.

McAllister et al. ${ }^{(20)}$ en la versión inglesa de la escala optaron por un análisis factorial exploratorio (AFE) que condujo, en primera instancia, a una solución de dos factores que consideraron poco satisfactoria dado que el segundo factor solo explicaba el $13,9 \%$ de la varianza, por lo que optaron por una solución con un único factor que proporcionaba una fiabilidad satisfactoria $(0,83)$. No aportan datos de la varianza explicada de dicha escala. La escala mantiene los 9 ítems originales, a pesar de que el ítem 1 ("creo que entiendo el problema que me llevo a Consejo Genético") tiene una carga factorial inferior a 0,30. El grupo de McAllister criticó las aportaciones del grupo de Shiloh, en cuanto a las puntuaciones obtenidas, señalando que el AFC no se comparó con otras posibles soluciones, y que nunca se valoró la existencia de otra posible estructura factorial.

Una posible explicación de las diferencias entre estos autores radicaría en el hecho de fundamentar los constructos en un modelo teórico concreto (caso del grupo de Shiloh) o no hacerlo. En la medida en que el análisis factorial confirmaba la estructura teórica, Shiloh y sus colaboradores consideraron innecesario plantear otras soluciones factoriales. Sin embargo, los otros autores optaron por una validez de constructo desligada de un modelo teórico específico y por ello optaron por un análisis exploratorio que permitiera obtener la mejor solución desde el punto de vista psicométrico. Ambos enfoques son justificables, pero en nuestra investigación hemos optado por el análisis exploratorio ya que consideramos que el control percibido puede ser perfectamente un constructo unidimensional, tal y como sugieren algunos trabajos realizados en el ámbito psicooncológico ${ }^{(13,28)}$. Además, el análisis factorial exploratorio es un método robusto y fiable para determinar la validez de constructo $^{(29)}$ que es la que pretendemos conseguir con la presente investigación. Finalmente, Si consideramos el número de ítems, observamos que nuestra versión reduce a seis ítems la escala original, sin por ello dejar de mostrar una estructura sólida y consistente, lo cual puede tener una relevancia práctica importante ya que se requerirá menor tiempo a las pacientes para responder el cuestionario.

Asumiendo que la escala cumple satisfactoriamente los requisitos psicométricos, falta establecer cómo interpretar las puntuaciones que se obtienen en la misma. A partir de los valores que facilitan los autores que han utilizado la solución unifactorial, podemos decir que estos oscilan entre el más bajo de 1,31 hallado por Bredart et al. y Albada et al. ${ }^{(27,30)}$ y el más alto de 1,45 hallado por Berkenstadt et al. ${ }^{(21)}$. En nuestra muestra, el valor medio hallado es de 1,32, no existiendo diferencias entre las PA y PS por lo que podemos concluir que la puntuación obtenida es independiente del hecho de ser pacientes oncológicos. Sin embargo, no se dispone de un criterio claro para considerar cuándo un paciente tiene un nivel de percepción de control "alto", "medio" o "bajo", ya que ni el grupo de Shiloh ni los otros equipos que han utilizado su escala lo han proporcionado. 
Para resolver esta cuestión proponemos un criterio basándonos en un trabajo previo de Juan et al. ${ }^{(31)}$ que estudió la relación entre percepción de control y supervivencia en pacientes de cáncer de pulmón. En dicha investigación se constató que las puntuaciones en percepción de control de los pacientes se situaban en la mayor parte de casos en los valores más altos de la escala utilizada, por lo que la variabilidad de las puntuaciones se restringía a un rango pequeño y de valores elevados. En base a ello, proponemos establecer un punto de corte en nuestra escala PPC6 que se sitúe en la zona de máxima puntuación y distinga entre las pacientes que tendrían una percepción de control "Normal" (es decir puntuaciones situadas en la zona media-alta de la escala) y pacientes con percepción de control "Alta" (es decir, puntuaciones situadas en la zona alta de la escala). La determinación de ese punto de corte se ha realizado en base al valor marcado por una desviación estándar por encima del valor promedio. Dado que el valor promedio encontrado es de 1,31 y la SD es de 0,53 consideraremos que el punto de corte se sitúa en 1,8 y, por tanto, la PPC6 puede interpretarse en base a dos niveles: percepción de control "Normal", para aquellos casos con valor inferior a 1,8 y percepción de control "Alta" para aquellos casos con valor igual o superior a 1,8 .

Nuestro trabajo tiene algunas limitaciones que deben ser tenidas en cuenta a la hora de extrapolar los resultados obtenidos, así como el punto de corte propuesto a partir de los mismos. Por un lado, la validez se ha realizado a partir de una muestra compuesta exclusivamente por mujeres con un diagnóstico muy concreto, por lo que es necesario que los resultados de nuestro trabajo sean replicados en otras muestras de pacientes pertenecientes a nuestro ámbito cultural y sometidos a consejo genético oncológico para otros tumores. Por otro lado, la muestra estaba compuesta únicamente por los datos de las pacientes que habían remitido por correo la batería de cuestionarios dentro del período de tiempo que podríamos considerar óptimo. Si bien esta tasa de respuesta es similar a la que se encuentra en otras investigaciones que utilizan el envío de datos mediante correo, no podemos asegurar que nuestra muestra no esté sesgada y que las pacientes que no remitieron la batería de cuestionarios dentro de las tres primeras semanas puedan tener un nivel y estructura de la percepción de control diferentes a los de las pacientes valoradas en la presente investigación.

A partir de estas consideraciones, estimamos, no obstante, que las posibilidades de aplicación clínica de la escala PPC6 son muy prometedoras, ya que es de fácil y rápida administración y permite identificar si la percepción de control que muestran las pacientes sometidos a consejo genético por cáncer de mama u ovario es la adecuada o, por el contrario, es un aspecto al que los equipos asistenciales presten atención a la hora de proporcionar a los pacientes el asesoramiento y los recursos necesarios para que el proceso de Consejo Genético proporcione resultados óptimos.

\section{Agradecimientos}

Este estudio ha sido posible gracias al soporte del Fondo de Investigación Sanitaria Proyecto PI10/00748 y PI13/00189 y de la Junta de Barcelona de la Asociación Española Contra el Cáncer. 


\section{Referencias bibliográficas}

1. Llort G, Serrano RMR, Sanchez AB, Teule A. SEOM clinical guidelines in Hereditary Breast and ovarian cancer. Clin Transl Oncol 2015;17:956-61. doi: 10.1007/s12094015-1435-3

2. Peshkin BN, Lerman C. Genetic counselling for hereditary breast cancer. Lancet 1999;353:2176-7. doi: 10.1016/j.gore.2015.10.001

3. Nelson HD, Fu R, Goddard K, Mitchell JP, Okinaka-Hu L, Pappas M, et al. Risk assessment, genetic counseling, and genetic testing for BRCA-related cancer: Systematic review to update the U.S. Preventive Services Task Force Recommendation. 2013;(101). doi: $10.7326 / \mathrm{M} 13-1684$

4. Den Heijer M, Seynaeve C, Vanheusden K, Timman R, Duivenvoorden HJ, TilanusLinthorst M, et al. Long-term psychological distress in women at risk for hereditary breast cancer adhering to regular surveillance: A risk profile. Psychooncology 2013;22:598-604. doi: $10.1002 /$ pon.3039

5. Ringwald J, Wochnowski C, Bosse K, Giel KE, Schäffeler N, Zipfel S, et al. Psychological distress, anxiety, and depression of cancer-affected BRCA1/2 mutation carriers: a systematic review. J Genet Couns 2016; 25: 880-91 doi: 10.1007/s10897-016-9949-66.

6. González M, Blanco I, Bayes R. Dificultad en la comunicación del riesgo de padecer cáncer hereditario. Psicooncología 2005; 2: 285-92. [Acceso el 15 de enero de 2018] Disponible en: http://diposit.ub.edu/dspace/handle/2445/44545

7. González M, Blanco I, Blasco T. Efectos de la primera visita de consejo genético sobre la percepción de riesgo y el malestar emocional. 2011; 8: 441-51. doi: 10.5209/rev_ PSIC.2011.v8.n2-3.37891

8. Cardenal C, Cruzado JA. Estrategias de afrontamiento y malestar emocional en cribado. Psicooncología 2014; 11: 285-99. doi: 10.5209/rev_PSIC.2014.v11.n2-3.47389

9. Castejónn V, Rovira T, Sumalla EC, Darder E, Iglesias S, Ochoa C, et al. Adaptación cultural y validación de la versión española de la Escala de Autoconcepto en BRCA en mujeres portadoras con alto riesgo de cáncer de mama y ovario hereditario. Med Clin (Barc) 2016;146:148-54. doi: 10.1016/j.medcli.2015.09.018

10. Pieterse AH, Ausems MGEM, Spreeuwenberg P, van Dulmen S. Longer-term influence of breast cancer genetic counseling on cognitions and distress: Smaller benefits for affected versus unaffected women. Patient Educ Couns 2011;85:425-31. doi: 10.1016/j. pec.2011.01.017.

11. Cruzado J, Perez P, Olivera H. Consecuencias y necesidades de la intervencion psicológica en consejo genético para mujeres en riesgo de cáncer de mama hereditario. Psicooncología 2007;4:465-82.

12. Vos J, Oosterwijk JC, Gomez-Garcia E, Menko FH, Collee MJ, van Asperen CJ, et al. Exploring the short-term impact of DNA-testing in breast cancer patients: the counselees' perception matters, but the actual BRCA1/2 result does not. Patient Educ Couns 2012;86:239-51. doi: 10.1016/j.pec.2011.04.017

13. Bárez M, Blasco T, Fernández-Castro J, Viladrich C. Perceived control and psychological distress in women with breast cancer: A longitudinal study. J Behav Med 2009;32:18796. doi: 10.1007/s10865-008-9180-5.

14. Hudson JL, Bundy C, Coventry PA, Dickens C. Exploring the relationship between cognitive illness representations and poor emotional health and their combined association with diabetes self-care. A systematic review with meta-analysis. J Psychosom Res 2014;76:265-74. doi: 10.1016/j.jpsychores.2014.02.004 
15. Bratzke LC, Muehrer RJ, Kehl KA, Lee KS, Ward EC, Kwekkeboom KL. Self-management priority setting and decision-making in adults with multimorbidity: A narrative review of literature. Int J Nurs Stud 2015; 52: 744-55. doi: 10.1016/j.ijnurstu.2014.10.010.

16. Shiloh S, Berkenstadt M, Meiran N, BatMiriamKatznelson M, Goldman B. Mediating effects of perceived personal control in coping with a health threat: The case of genetic counseling. J Appl Soc Psychol 1997; 27: 1146-74. doi: 10.1111/j.1559-1816.1997. tb01799.x

17. Davey A, Rostant K, Harrop K, Goldblatt J, O’Leary P. Evaluating genetic counseling: Client expectations, psychological adjustment and satisfaction with service. $\mathrm{J}$ Genet Couns 2005;14:197-206. doi: 10.1007/s10897-005-0519-6

18. Robinson CL, Jouni H, Kruisselbrink TM, Austin EE, Christensen KD, Green RC, et al. Disclosing genetic risk for coronary heart disease: effects on perceived personal control and genetic counseling satisfaction. Clin Genet 2016; 89: 251-7. doi: 10.1111/cge.12577

19. Smets EMA, Pieterse AH, Aalfs CM, Ausems MGEM, Dulmen AM Van. The Perceived Personal Control (PPC) Questionnaire as an outcome of genetic counseling: Reliability and validity of the instrument. Am J Med Genet A 2006; 40:843-50. doi: 10.1002/ ajmg.a.31185

20. McAllister M, Wood AM, Dunn G, Shiloh S, Todd C. The perceived personal control (PPC) questionnaire: reliability and validity in a sample from the United Kingdom. Am J Med Genet A 2012; 158:367-72. doi: 10.1002/ajmg.a.34374.

21. Berkenstadt M, Shiloh S, Barkai G, Katznelson MBM, Goldman B. Perceived personal control (PPC): A new concept in measuring outcome of genetic counseling. Am J Med Genet A 1999;82:53-9. doi: 10.1002/(SICI)1096-8628(19990101)82:1<53::AIDAJMG11>3.0.CO;2-\#

22. Cha ES, Kim KH, Erlen JA. Translation of scales in cross-cultural research: Issues and techniques. J Adv Nurs. 2007;58:386-95. doi: 10.1111/j.1365-2648.2007.04242.x

23. Chen HY, Boore JRP. Translation and back-translation in qualitative nursing research: Methodological review. J Clin Nurs 2010;19:234-9. doi: 10.1111/j.13652702.2009.02896.x.

24. Beranuy M, Chamarro A, Graner C, Carbonell X. Validación de dos escalas breves para evaluar la adicción a Internet y el abuso de móvil. Psicothema 2009;21:480-5.

25. Averill JR. This Week's Citation Classic. 1983;1983.

26. Aalfs CM, Oort FJ, de Haes JCJM, Leschot NJ, Smets EM a. A comparison of counselee and counselor satisfaction in reproductive genetic counseling. Clin Genet 2007;72:74-82. doi. 10.1111/j.1399-0004.2007.00834.x

27. Brédart A, Kop JL, Depauw A, Caron O, Sultan S, Leblond D, et al. Short-term psychological impact of the BRCA1/2 test result in women with breast cancer according to their perceived probability of genetic predisposition to cancer. Br J Cancer 2013;108:1012-20. doi: 10.1038/bjc.2012.599

28. Bárez M, Blasco T, Fernandez-Castro J, Viladrich C. Need for cognition and psychosocial adjustment in prostate cancer patients and partners. J Psychosoc Oncol 2007;25:37-41. doi; 10.1300/J077v25n01_01

29. Letelier MJ, Aller MB, Henao D, Sánchez-Pérez I, Vargas Lorenzo I, Coderch de Lassaletta $\mathrm{J}$, et al. Diseño y validación de un cuestionario para medir la continuidad asistencial entre niveles desde la perspectiva del usuario: CCAENA. Gac Sanit 2010;24:339-46. doi: $10.1016 /$ j.gaceta. 2010.03 .010 
30. Albada A, Ausems MGEM, van Dulmen S. Counselee participation in follow-up breast cancer genetic counselling visits and associations with achievement of the preferred role, cognitive outcomes, risk perception alignment and perceived personal control. Soc Sci Med 2014;116:178-86. doi: 10.1016/j.socscimed.2014.07.012

31. Juan E, Blasco T, Font A, Doval E, Sanz A, Maroto P. y Pallarés C. Percepción de control y superviviencia en pacientes con cáncer de pulmón avanzado sometidos a tratamiento paliativo. Ansiedad y Estrés 2003; 9:1-5. 